ORIGINAL ARTICLE

\title{
Exosomes derived from chronic myeloid leukemia cells: roles in disease progression, survival, and treatment
}

\author{
SAYEH PARKHIDEH PHD ${ }^{\dagger}$, MAHSHID MEHDIZADEH M.D ${ }^{1}$, ABBAS HAJIFATHALI M.D ${ }^{1}$, HANIYEH GHAFFARI NAZARI \\ $M S^{1 \dagger}{ }^{1 \dagger}$, ELHAM ROSHANDEL P.H.D ${ }^{1^{*}}$, REZA MIRFAKHRAIE ${ }^{*}$ P.H.D ${ }^{1}$ \\ ${ }^{1}$ Hematopoietic Stem Cell Research Center, Shahid Beheshti University of Medical Sciences, Tehran, Iran \\ Correspondence: Elham Roshandel \& Reza Mirfakhraie (email: elham.roshandel@gmail.com) \\ Hematopoietic Stem Cell Research Center, Shahid Beheshti University of Medical Sciences, Tehran, Iran \\ Postal code/ P.O. Box: 1985711151, Tel \#: +98 21 22432560, Fax \#: +98 21 22432560, Mobile phone \#: +98 9121782734
}

\begin{abstract}
Exosomes, biologically active extracellular vesicles, are derived from normal and neoplastic cells. Emerging evidence revealed that exosomes modulate cell-cell communication and involve in hemostatic and pathologic processes. Recent studies have shown that exosomes released from cancer cells such as chronic myeloid leukemia cells could act as a key mediator in tumor induction and progression. Myeloid cells-derived exosomes affect different processes including angiogenesis, neoplastic proliferation, tumor cell survival, and imatinib resistance. These exosomes induce angiogenesis and tumor progression by IL-8 overexpression in both leukemic and bone marrow stromal cells. Exosomes cargo could alter the expression of different adhesion molecules, antiand pro-apoptotic molecules, cytokines, and chemokines such as VCAM-1, ICAM-1, BCL, BAD, BAX, TGF- $\beta$, TNF- $\alpha$, CXCL12 which affect tumor migration, homing, survival, and growth. CML-derived exosomes can also regulate signal transduction pathways, such as ERK/MAPK, ERK/Akt, EGFR/Ras, and Wnt. Furthermore, they can be applied as a vehicle for drug delivery or sensitization of drug-resistant cells. Here, we reviewed the role of chronic myeloid cell-derived exosomes in tumor growth, survival, and resistance to treatment.
\end{abstract}

Keywords: Chronic myeloid leukemia, Exosome, Angiogenesis, Tyrosine kinase inhibitor, Drug resistance.

\section{INTRODUCTION}

Chronic myeloid leukemia (CML), a myeloproliferative neoplasm, accounts for approximately $15 \%$ of all cases of leukemia diagnosed in adults (1). CML is caused by an oncogenic translocation of $\mathrm{t}(9 ; 22)(\mathrm{q} 34 ; \mathrm{q} 11)$ known as Philadelphia chromosome $(\mathrm{Ph})$ resulting in expression of the BCR-ABL fusion protein (2). BCR-ABL promotes DNA replication and cellular proliferation through activating tyrosine kinase and downstream signaling molecules including RAS, RAF, JUN kinase, MYC, and STAT (3-5). Activated BCR-ABL also alters cell adhesion and inhibits apoptosis (6).

This triphasic disorder begins from a latent phase named chronic phase (CML-CP), the second phase refers to its spontaneous progression to advanced accelerated phase (CML-AP) in untreated patients, and the third phase indicates CML development to aggressive blast crisis phase (CML-BP) (7). CML is diagnosed based on the presence of persistent unexplained leukocytosis, and the detection of the $\mathrm{Ph}$ chromosome, $\mathrm{t}(9 ; 22)(\mathrm{q} 34 ; \mathrm{q} 11)$, or $\mathrm{Ph}$ related molecular BCR-ABL1 abnormalities by cytogenetics, fluorescence in situ hybridization (FISH), and molecular analysis (8). However, there are some challenges in CML detection; for example difficulties in the diagnosis of $\mathrm{Ph}$-negative $\mathrm{CML}$ cases and differential diagnosis of CML from other myeloproliferative or myelodysplastic syndromes (8).

The emergence of tyrosine kinase inhibitors such as imatinib, nilotinib, dasatinib, bosutinib and ponatinib has significantly decreased the mortality rate from $10 \%-20 \%$ to $1 \%-2 \%$ and has prolonged the overall survival in $\mathrm{CML}$ patients. Nonetheless, BCR-ABL gene amplification or secondary point mutations in tyrosine kinase domain of BCR-ABL may develop resistance to imatinib in some patients (8).
Understanding molecular mechanisms involved in the disease process and progression may provide novel approaches to develop anti-cancer drugs and therapies. Recent studies have revealed that the crosstalk between cancer cells and their microenvironment could regulate the growth, survival, and drug-resistance of leukemic cells. Recent evidence has shown that cancer cells could release extracellular vesicles such as exosomes which modulate the interaction between tumor cells and their microenvironment (9).

Exosomes are lipid vesicles with endosomal origin and mean diameters ranging from $40 \mathrm{~nm}$ to $160 \mathrm{~nm}$. Nowadays, exosomes are considered to serve as potential novel cellular signaling elements, which are secreted by various cells (normal and tumor cells) and could regulate intercellular communications. They can mediate autocrine, juxtacrine and paracrine interactions. These extracellular vesicles could be isolated from bio fluids samples; for instance blood, lymph fluid, cerebrospinal fluid (CSF), saliva, and urine (10). Exosomes are enriched in distinct molecular cargo such as signaling receptors and ligands in the membrane along with proteins (cytoplasmic, nuclear, extracellular matrix), lipids, metabolites, and nucleic acids (DNAs, messenger RNA (mRNAs), microRNAs, long noncoding RNAs (IncRNAs), circularRNA, etc) within the cytoplasmic matrix (11). Exosomes contents represent their cell of origin and can affect biological responses in recipient cells. In context of cancer development, exosomes derived from the tumor cells and tumor microenvironment (TME) such as endothelial cells, pericytes, fibroblasts, mesenchymal stem cells (MSCs) and immune cells could modulate the cross talk between tumor and microenvironment $(12,13)$. For example, Ramos et al. found that the endothelial cells can acquire BCR-ABL RNA of $\mathrm{Ph}+$ positive $\mathrm{CML}$-derived exosomes that is inconsistent with the hypothesis that endothelial cells may be part of the 
CML clone (14). Therefore, exosomes play an integral role in the induction or promotion of tumor growth, metastasis, relapse, drug response or resistance, and disease mechanism. Nowadays, exosomes appear to be interesting biomarkers for cancer diagnosis, cancer prognosis, staging, progression, and drug response. Bernardi et al. reported that the CML-derived exosomes could be possibly enriched and considered as novel biomarkers to diagnose active leukemic cells in patients. The miRNA content of exosomes derived from CML cells may be associated with patients' clinical symptoms such as cancer-associated cachexia, and musculoskeletal pain. The elevation of exosomal miRNA-140-3p serum levels after tyrosine kinase inhibitors discontinuation and the overexpression of exosomal miR-92a-3p might lead to musculoskeletal pain and cachexia in $\mathrm{CML}$ patients, respectively $(15,16)$. Other studies have demonstrated that the exosomes released from CML cells have the ability to affect bone marrow niche and tumor microenvironment (17). According to this evidence, here we reviewed the impact of CML-derived exosomes in CML pathogenesis, progress, and treatment.

CML- derived exosomes and angiogenesis: Although the detection of BCR-ABL expression is enough for CML chronic phase diagnosis, additional mechanisms are required for progression to aggressive phases and resistance to treatment. Emerging evidences indicate significant neovascularization and an increased number of endothelial cells in bone marrow of CML patients (18). Therefore, it seems that angiogenesis plays an essential role in the development and progression of $\mathrm{CML}$. However, little is known about mechanisms responsible for angiogenesis in CML. New blood vessels formation provides oxygen and nutrition for cancer cells and promotes tumor cell's survival, growth, and distant metastasis development. Angiogenesis depends on a balance of pro-and anti-angiogenic factors such as vascular endothelial growth factor (VEGF), fibroblast growth factor-2 (FGF-2), hepatocyte growth factor (HGF), interleukin-8 (IL-8), matrix metalloproteinases (MMPs), platelet factor-4 (PF-4), and tissue inhibitors of metalloproteinases (TIMPS)) molecules released by tumor cells. Increased levels of angiogenic factors such as IL-8 have been detected in several myeloid and lymphoid haematological malignancies $(19,20)$. It is shown that BCR-ABL oncogene contributes to VEGF- mediated angiogenesis through activating the PI3-kinase, AKT, mTOR and HIF-1a pathways. The functional role of exosomes in tumor progression has yet to be elucidated, but investigations have indicated the importance of CMLderived exosomes in regulating crosstalk between leukemic and stromal cells in tumor microenviroment and their direct effect on endothelial cells by modulating neovascularization.

Taverna et al. isolated and characterized the exosomes released from LAMA84, a human CML cell line, for the first time. They demonstrated that treating human vascular endothelial cells (HUVECs) with CML cells-derived exosomes could stimulate in vitro angiogenesis related functions including motility, cytokine production, cell adhesion and cell signaling, as well as angiogenesis induction in a nude mice model. LAMA84-derived exosomes increased IL-8 expression in the HUVECs. In addition, endothelial cells stimulated by exosomes enhanced mRNA level of cell-cell adhesion molecules, VCAM-1 and ICAM-1, expression in a dose- and timedependent manner (21). VCAM-1 and ICAM-1 are expressed by cytokine-activated endothelial cells during the inflammatory process (22). IL-8 neutralization, however, could inhibit LAMA84 exosome-stimulated increase of ICAM-1 and VCAM- 1 in HUVECs. LAMA84 exosomes could also mediate cell migration process, which is critical for angiogenesis, via affecting VE-cadherin and $\beta$ catenin expression. The linkage of VE-cadherin to the cytoskeleton is critical for adherens junction organization and strong cellcell interaction (23). VE-cadherin expression reduction and the translocation of $\beta$ catenin from the plasma membrane to the cytoplasm and nucleus was detected in exosome treated HUVECs. Loss of VE-cadherin and alteration of $\beta$ catenin distribution could affect the maintenance of adherens junction and promote permeability, proliferation, activation, mobilization of endothelial cells, and angiogenesis (24). Additional analysis revealed that the exosomes might regulate signal transduction in HUVECs by inducing the phosphorylation of extracellular signalregulated kinase $(E R K) /$ mitogen-activated protein kinase (MAPK) signaling pathway. Indeed, Exosomes released from CML cells could activate signal transduction pathways leading to IL-8 production with active angiogenic phenotype (24). It was demonstrated that the exosomes released by K562 CML cells can promote vascular differentiation and angiogenesis through focal adhesion kinase (FAK), a substrate of Src kinase, phosphorylation and its downstream pathway's activation in HUVECs (25). FAK mediates both normal and tumor cell migration and correlates to several signaling pathways such as the ERK2/MAPK cascade and Rho-family GTPases. FAK-Src kinase disassembles focal adhesions via activating intracellular proteases such as calpain and extracellular matrix metalloproteinases (26) and regulates cadherinmediated cell-cell contacts. Recent findings also revealed that the exosomes derived from $\mathrm{K} 562 \mathrm{CML}$ cells could induce neo-angiogenesis in the chorioallantoic membrane by increasing VEGFR1 expression (27).

MiRNAs, as a part of exosome cargo, can control gene expression, regulate physiological function, and induce pathological processes in target cells (28). There are evidences suggesting that exosomal miRNAs', derived from the K562 cells, have putative role in metabolism, cell cycle, cell adhesion, angiogenesis, tumorigenesis processes and $\mathrm{CML}$ progression $(29,30)$. Feng et al showed up-regulation of 49 miRNAs in K562 cells-derived exosomes. Bioinformatical analysis, Gene Ontology, and KEGG pathway analysis indicated that miRNAs upregulation in exosomes may be associated with Wnt signaling pathway, cell adhesion, cell migration, negative regulation of apoptotic process and Wnt receptor signaling pathway, positive regulation of mesenchymal cell proliferation and cell proliferation. They found several CML exosomal miRNAs including; miR-3646, miR-298, miR-2995p, miR-1827, miR-665, miR-4268, miR-4290, miR-483-3p, miR-498, miR-525-5p, miR-3611, miR-3686, miR-711, miR423-5p, miR-4279, miR-3915, miR-1973, miR-3201, miR612 , miR-943 and miR-518b which might modulate the Wnt 
signaling pathway and promote the proliferation, migration, angiogenesis, invasion, and metastasis of $\mathrm{CML}$ cells via manipulating the microenvironment (29). In hypoxic conditions, K562 CML cells secrete exosomes with high levels of miR-210 that could enhance tube formation in HUVECs. MiR-210 targets the receptor tyrosine kinase ligand, Ephrin-A3 (EFNA3), and down-regulates its expression. The regulatory role of EFNA3 in angiogenesis is still unclear. It seems that, EFNA1-EphA2 interaction could potentially regulate VEGF signaling and angiogenesis. Since EphA2 can also bind to EFNA3, it might act as a negative regulator for angiogenesis. Therefore, high exosomal miR-210 expression would enhance HUVECs tube formation through EFNA3 downregulation (30). The miR-17-92a cluster in K562-derived exosomes, especially miR-92a, has been known as an angiogenesis regulator. Exosomal miR-92a internalize endothelial cells and enhance their migration and tube formation by reducing integrin a5 protein expression (31). LAMA84 CML-derived exosomes could also transfer MiR126 that plays a critical role in angiogenesis. MiR-126 in exosomes secreted from LAMA84 CML cells, could be transferred to endothelial cells and down-regulate the expression of CXCL12 and VCAM1through targeting the 3' UTR region of their mRNA. CXCL12 and VCAM1 are two important components in bone marrow niche $(32,33)$. Some studies have suggested that the modulation of CXCR4/CXCL12 chemotaxis gradient is likely to be associated with CML blastic cells migration to the circulation. Taverna et al. have hypothesized that in early stages of blastic cells migration, exosomal miR-126 induces VCAM1 expression in endothelial cells to attach leukemic cells on the endothelium. During the late stage, following a prolonged exposure of endothelial cells with exosomes, the expression of CXCL12 and VCAM1 will reduce, and leukemic cells will migrate towards a richer source of chemo attractants (21). Exosomal cargo would be regarded as a linkage between $\mathrm{CML}$ cells and other cells in tumor microenvironment, although the functional mechanisms have not yet been clarified completely. According to aforementioned studies, the critical role of IL-8 in modulating $\mathrm{CML}$ progression by $\mathrm{CML}$-derived exosomes can never be overstated. IL-8 mediates paracrine effects that affect leukemic cells and their microenvironment. Furthermore, the expression of various adhesion molecules, and chemokines as well as different signal transduction pathways, which are contributed to angiogenesis and cell migration, can alter the behavior of tumor cells and their microenvironment, and promote tumor progression via exosomal cargo.

CML-derived exosomes and leukemic cells proliferation and survival: In the context of tumor growth, sustained activation of several molecular mechanisms in tumor cells causes tumor growth and survival. For instance, these mechanisms can lead to tumor cell resistance to apoptosis or tumor escape from immune response that appears to favor tumor progression (34, 35). Recent investigations have suggested that the tumor cells-derived exosomes could involve in tumor cell proliferation, growth, and survival through activating signal transduction pathways. CML cells exposure to the exosomes derived from LAMA84-culture might promote proliferation and colony formation in a dose-dependent manner. Furthermore, CML xenograft mouse models treated with LAMA84-derived exosomes demonstrated greater tumor size than those in the control group without any treatments. Real time PCR and western blot analysis demonstrated an increase in BCL-w, BCL-xl, and survivin anti-apoptotic molecules and a reduction in BAD, BAX, and PUMA proapoptotic molecules as a result of $\mathrm{CML}$ cells stimulation with exosomes (36). CML exosomes are enriched with transforming growth factor $\beta 1$ (TGF- $\beta 1$ ), that is a multifunctional cytokine with various functions such as controlling cell growth, cell proliferation, cell differentiation, and apoptosis. TGF- $\beta 1$ signaling inhibition might suppress the exosome-induced anti-apoptotic phenotype of CML cells (36). Therefore, the interaction between TGF- $\beta 1$ enriched exosomes and TGF- $\beta 1$ receptors on CML cells could lead to cells proliferation and survival by activating anti-apoptotic pathways (37). Moreover, CML-derived exosomes can stimulate ERK and Akt phosphorylation and NF-kB activation in LAMA84 leukemic cells. The PI3/Akt or MAPK/ERK pathways are likely to control cancer cells growth and survival (38). NF-kB is a major transcription factor that regulates genes responsible for survival, apoptosis, and immune response while interacting with different signaling pathways such as PI3/Akt (39). CML cells derived exosomes are able to promote leukemic cell proliferation and survival by stimulating direct autocrine effects and cell growth signal transduction pathways, and inhibiting apoptotic pathways (36). Such exosomes are also thought to activate bone marrow (BM) stromal cells to produce IL-8 and stimulate an IL 8-mediated autocrine loop in BM stromal cells both in vitro and in vivo. Exosomes released from $\mathrm{CML}$ cells appeared to modulate $\mathrm{IL}-8$ expression in bone marrow microenviroment. It is confirmed that the receptors of IL-8, CXCR1 and CXCR2, are expressed on $\mathrm{CML}$ cells. IL-8 regulates $\mathrm{CML}$ cells behavior in BM microenvironment and enhances malignant cells survival through their adhesion to BM stroma. Signal transduction pathways analysis has indicated the impact of IL-8 in Akt phosphorylation induction in CML cells which promotes leukemic cells survival due to $\mathrm{CML}$ cells derived exosomes stimulation (40). Leukemic cells could also produce exosomes containing PAK2 (p21-activated kinases) as an upstream regulator of STAT5 transcription factor in BCR/ABL1+ cells. Exosomes containing PAK2 protein, along with other PAK isoforms, play essential role in disease initiation, maintenance, and endothelial proliferation (41).

CML-derived exosomes and bone marrow niche: The bone marrow niche is a local tissue microenvironment that supports and regulates self-renewal of normal and malignant hematopoietic cells. BM niche is comprised of several cellular compositions including; MSCs, endothelial cells, osteolineage cells, and hematopoietic stem cell (HSCs) progeny (42). As mentioned before, a cross talk between BM niche and leukemic cells is essential for cancer progression and drug resistance (43). Exosomes released from CML cells acquire the ability to remodel a normal hematopoietic niche to a tumor-supportive microenvironment. Corrado and colleagues have found that CML cells-derived exosomes could stimulate amphiregulin (AREG)-epidermal growth factor receptor (EGFR) axis in 
BM stromal cells and induce IL8 secretion. LAMA84 and CML cells-derived exosomes contain amphiregulin protein, which is an EGFR ligand with an ability to activate EGFR signaling pathway in stromal cells and involves in apoptosis, angiogenesis, tissue invasion, and metastasis (44). EGFR has some downstream targets that induce several cytokines or growth factors production and regulate cell proliferation, migration and apoptosis (45). SNAIL, a downstream target of EGFR, activation following EGFR signaling will regulate IL-8 and MMP9 expression in stromal cells $(44,46)$. Furthermore, the increase in annexin A2 expression in BM stromal cells after CML cells derived exosomes release could promote leukemic cells adhesion to BM stromal layer, tumor growth and invasion. Annexin A2 contributes to various cancer progression processes including cell proliferation, adhesion, migration, invasion, and angiogenesis. Moreover, this protein modulates EGFR/Ras pathway indirectly in association with different signal transduction molecules such as Src tyrosine kinases, Rho, Cdc42, Ras and GTPase activating protein (GAP) (44). K562 cells-derived exosomes can affect bone marrow-derived mesenchymal stem cells (BM-MSCs) and macrophages in providing a supportive immunosuppressive microenvironment for leukemic cells. These exosomes would alter the expression of CXCL2, Dickkopf-related protein 1 (DKK1), Wnt5a, and TNF-alpha as well as the production of nitric oxide (NO) and reactive oxygen species (ROS) in BM-MSCs and macrophages (47). Exposure to CML cells-derived exosomes can down-regulate CXCL12 expression in BM-MSCs. BM resident MSCs produce CXCL12 chemokines that support the hematopoietic cells' function and maintenance. It seems that CXCL12 reduction after the exposure to exosomes will decrease the number of normal HSCs and promote CML leukemic cells growth. Moreover, high CXCL12 expression in BM-MSCs may induce drug resistance by protecting leukemic stem cells against drug-induced apoptosis. CML cells-derived exosome dysregulates the expression of Wnt signaling inhibitors including Wnt5a and DKK1 and induces oncogenic effects $(48,49)$. DKK1 and Wnt5a could indirectly support the immunosuppressive effects, accumulation of myeloid-derived suppressor cells (MDSCs), cell proliferation and migration via $\beta$-catenin down-regulation $(50,51)$. TNF- $\alpha$ is another cytokine in a leukemic niche that significantly increases in both BMMSCs and macrophages after treatment with K562-derived exosomes. It has been shown that the presence of TNF- $\alpha$ in the BM provides an inflammatory niche to support the proliferation and maintenance of stem cells and progenitor cells (52), angiogenesis, extra-medullary infiltration, immune suppression, and drug resistance in CML and other hematologic malignancies (53). CML cells-derived exosomes can also impair redox balance (oxidationreduction reactions) in the macrophage and BM-MSC in favor of tumor-promoting microenvironment. Ren et al. have reported the suppressive effect of BM-MSCs in cell proliferation and immune function by elevating $\mathrm{NO}$ production (54). Furthermore, an increased level of NO has been detected in CML patient's serum (55). NO increases in BM-MSCs after exposure to exosomes, therefore, may attribute to immunomodulation, angiogenesis, cellular function, and tumor behavior. In addition, exosome- induced TNF- $\alpha$ and NO production may direct macrophages polarization toward tumor-associated M2-like (TAM2) phenotype (56). Gao et al. have declared that in CML chronic phase mouse model, the miRNA content of CML cells-derived exosomes would affect BM niche by inhibiting osteogenesis and promoting leukemic cells proliferation both in vitro and in vivo. They showed that miR-320 family, within CML cells-derived exosomes, are involved in leukemic niche remodeling (57). MiR-320 family are tumor suppressor miRNAs that can target the $\mathrm{BCR} / \mathrm{ABL}$ oncogene and $\beta$-catenin directly, and contain the AGAGGG motif which may control their exosomal sorting (58). This family can influence both leukemic cells and adjacent resident cells in BM niche including BM-MSCs. Transfection of K569 CML cells with miR-320a reduces endogenous $\mathrm{BCR} / \mathrm{ABL}$ expression and inhibits cell proliferation by arresting cell cycle at $\mathrm{G} 1$ phase. On the other hand, miR-320a can down-regulate $\beta$-catenin, a transcriptional regulator of canonical Wnt signaling, and stimulates osteogenesis. Blast crisis CML (CML-BC) patients have lower intracellular levels of miR-320 expression but higher plasma exosomal miR-320 levels than chronic phase CML (CML-CP) patients (57). Moreover, RNA-binding proteins ,HNRNPA1, responsible for exosomal RNA export and specific exosomal miRNA sorting, would be elevated in both CML-CP and CML-BC cells in mRNA and protein levels (59). Recent studies have proposed that the exosomal miR-320 might be transferred from leukemic cells to BM-MSC. This transportation is associated with miR-320's intracellular level decrease in leukemic cells and cancer cell proliferation. On the other hand, receiving exosomal miR-320 from BM-MSC causes $\beta$-catenin inhibition and osteogenesis (57). Various CMLderived exosomes effects on the disease progression are summarized in figure 1.

CML cells-derived exosomes and drug resistance: Imatinib resistance remains a significant complication of treatment for CML which develops in approximately $20 \%$ to $30 \%$ of the patients $(60,61)$. Accumulated evidence has identified exosomes that can induce drug resistance in target cells. Imatinib-resistant exosomes derived from $\mathrm{CML}$ cells could be transmitted to imatinib-sensitive CML cells and induce drug resistance in these cells. Analyzing exosomes' miRNA profile has confirmed the up-regulation of different miRNAs in drug resistant cells. Among these up-regulated miRNAs, miR-365a showed the highest expression level in imatinib resistant cells and imatinib resistant cells-derived exosomes. miR-365a inhibits cell apoptosis by down-regulating the expression of proapoptotic related proteins, BAX and CASPASE-3, and rendering $C M L$ cells resistant to imatinib-induced apoptosis (62).

Exosomes and their Therapeutic applications: Several immunomodulatory characteristics have been reported for tumor-derived exosomes. Therefore, it is suggested that exosomes can be used in cancer immunotherapy and other therapeutic strategies. Emerging evidence showed that exosomes containing tumor antigens can stimulate the antitumor immune responses (63). Phase I clinical trials in human melanoma and non-small cell lung cancer have proven the safety, feasibility, and immunogenicity of patient-specific exosomes. In this study, dendritic cells- 
derived exosomes loaded with tumor antigens could induce both innate and adaptive immune responses, disease control, and long-term survival in some patients (64). Exosomes may be potential candidates for vaccine design against infection, allergy, and autoimmune diseases (6567). These exosomes have also proven to be a valuable tool for the delivery of therapeutic agents such as antiinflammatory agents, RNA interference (siRNA), microRNA regulatory molecules, and other single-stranded oligonucleotides $(68,69)$. Dong et al. have evaluated therapeutic exosomes in CML and reported the ability of alkalized exosomes in sensitizing cells resistant to imatinib. Although the underlying mechanisms of imatinib resistance have not fully understood, some miRNA families such as miR-328 are suggested to be responsible in imatinib resistance. Lysosomal degradation of endogenous miR328 decrease its concentration and lead to imatinib resistance in $\mathrm{K} 562 \mathrm{CML}$ cells, subsequently. ABCG2 expression increases as a result of miR-328 expression reduction. A subfamily of ATP-binding cassette superfamily $G$ member proteins encoded by ABCG2 gene could transport molecules across cell membranes. ABCG2 overexpression reduces the intracellular concentration of imatinib when transfers to the outside of the cell. Therapeutic delivery of alkalized exosomes with or without miR-328, could block miR-328 degradation and sensitize cells resistance to imatinib (70). Another study revealed that curcumin is likely to play an antineoplastic role in an exosome-mediated manner. Treating CML cell lines with curcumin induces selective packaging of miR-21 in CMLderived exosomes that decreases miR-21 in cells, consequently. MiR-21 decrease may cause phosphatase and tensin homolog (PTEN) overexpression, an antagonist of the PI3K-AKT pathway, which in turn decreases AKT phosphorylation, suppresses PI3K/AKT signaling pathway, and down-regulates VEGF expression, leading to the inhibition of leukemic cell's growth and migration. Taken together, it seems that curcumin could potentially mediate antineoplastic effects via exosomal disposal of miR-21 (71).

Bellavia et al. have utilized exosomes as vehicles for specific drug delivery to the tumor site. Considering high expression of IL3 receptor (IL3-R) on CML blasts, they could target IL3-R for specific drug delivery. Firstly, HEK293T cell was engineered to express fused protein Lamp2b and a fragment of interleukin 3 (IL3-Lamp2b (IL3L)) as a source of exosome. Then, exosomes containing IL3L were loaded with either imatinib or BCR$A B L$ siRNA. In vitro analysis revealed that treatment of LAMA84 and K562 cell lines with both Imatinib- and BCRABL siRNA-loaded exosomes reduced viability of leukemic cells. In vivo study in LAMA84 -inoculated NOD/SCID mice model confirmed the efficacy of imatinib- and BCR-ABL siRNA-loaded exosomes in inhibiting tumor growth and reducing tumor size as compared to other groups. They also showed abundant IL3L exosomes accumulation in the tumor site short time after injection. Therefore, it is suggested that exosomes are able to be applied as a suitable vehicle for specific targeted therapy in CML (72).

\section{CONCLUSION}

CML cells communicate with other leukemic cells and tumor microenvironment cells such as bone marrow stromal cells, mesenchymal stem cells, endothelial cells, immune cells, etc. via exosomes. CML cells-derived exosomes can affect angiogenesis, leukemic cell proliferation, and survival as well as drug resistance. According to the above described studies, these exosomes' content can regulate different signal transduction pathways such as ERK/MAPK, Akt, Wnt, NFkB, and involve in disease induction and progression. However, most of the research studies have been conducted using in vitro cell culture-based systems. Therefore, it is essential to design experiments based on relevant physiological experimental conditions and CML animal models to clarify the exact biogenesis, trafficking, and homeostatic or pathological functions of exosomes. Although exosomes are potential tools for identification CML pathogenesis and treatment, further studies are needed for applying exosomes in clinical cancer diagnosis, staging, and therapy.

Acknowledgment: The authors would like to thank the staff of the Hematopoietic Stem Cell Research Center, Shahid Beheshti University of Medical Sciences, Tehran, Iran for providing the possibility of writing this manuscript.

Notes on contributors: S.P., M.M., H.G.N, A.H.; performed the literature search and manuscript drafted. and E.R., S.P; Supervision, idea suggestion Critical revision of the article. All authors revised the manuscript and approved the final paper. Conception or design of the work.

Funding: This research did not receive any specific grant from funding agencies in the public, commercial, or not-forprofit sectors.

Conflict of Interest: The authors declare no conflict of interest.

\section{REFERENCE}

1. Soverini S, De Benedittis C, Mancini M, Martinelli G. Best Practices in Chronic Myeloid Leukemia Monitoring and Management. Oncologist. 2016;21(5):626-33.

2. Goldman JM. Chronic Myeloid Leukemia: A Historical Perspective. Semin Hematol. 2010;47(4):302-11.

3. Raitano AB, Halpern JR, Hambuch TM, Sawyers CL. The BcrAbl leukemia oncogene activates Jun kinase and requires Jun for transformation. Proc Natl Acad Sci. 1995;92(25):11746-50.

4. Mandanas RA, Leibowitz DS, Gharehbaghi K, Tauchi T, Burgess GS, Miyazawa K, et al. Role of p21 RAS in p210 bcr-abl transformation of murine myeloid cells. 1993.

5. Okuda K, Matulonis U, Salgia R, Kanakura Y, Druker B, Griffin J. Factor independence of human myeloid leukemia cell lines is associated with increased phosphorylation of the protooncogene Raf-1. Exp Hematol. 1994;22(11):1111-7.

6. Jabbour E, Cortes JE, Ghanem H, O'Brien S, Kantarjian HM. Targeted therapy in chronic myeloid leukemia. Expert Rev Anticancer Ther. 2008;8(1):99-110.

7. Danilo Perrotti CJ, 2 John Goldman, 3 and Tomasz Skorski4. Chronic myeloid leukemia: mechanisms of blastic transformation. J Clin Invest. 2010. 120(7):2254-64.

8. Jabbour E, Kantarjian H. Chronic myeloid leukemia: 2020 update on diagnosis, therapy and monitoring. Am J Hematol. 2020;95(6):691-709.

9. Zhang C, Ji Q, Yang Y, Li Q, Wang Z. Exosome: Function and role in cancer metastasis and drug resistance. Technol Cancer Res Treat. 2018;17:1533033818763450.

10. Kalluri R, LeBleu VS. The biology function and biomedical applications of exosomes. Science. 2020;367(6478):eaau6977.

11. Yang C, Yang H, Liu J, Zhu L, Yu S, Zhang X, et al. Focus on exosomes: novel pathogenic components of leukemia. Am J Cancer Res. 2019;9(8):1815-29.

12. Pando A, Reagan JL, Quesenberry P, Fast LD. Extracellular vesicles in leukemia. Leuk Res. 2018;64:52-60. 
13. Ohyashiki JHU, Tomohiro 2 ; Ohyashiki, Kazuma 3 ; . Exosomes promote bone marrow angiogenesis in hematologic neoplasia: the role of hypoxia. Curr Opin Hematol. 2016;23:. 268-73(6).

14. Ramos TL, Sánchez-Abarca LI, López-Ruano G, Muntión S, Preciado S, Hernández-Ruano $M$, et al. Do endothelial cells belong to the primitive stem leukemic clone in CML? Role of extracellular vesicles. Leuk Res. 2015;39(8):921-4.

15. Wan Z, Chen X, Gao X, Dong Y, Zhao Y, Wei M, et al. Chronic myeloid leukemia-derived exosomes attenuate adipogenesis of adipose derived mesenchymal stem cells via transporting miR92a-3p. J Cell Physiol 2019;234(11):21274-83.

16. Asano M, Umezu T, Katagiri S, Kobayashi C, Tauchi T, Gotoh M, et al. Up-regulated exosomal miRNA-140-3p in CML patients with musculoskeletal pain associated with discontinuation of tyrosine kinase inhibitors. IntJ Hematol. 2017;105(4):419-22.

17. Ohyashiki JH, Umezu T, Ohyashiki K. Exosomes promote bone marrow angiogenesis in hematologic neoplasia: the role of hypoxia. Cur Opin Hematol. 2016;23(3):268-73.

18. Kvasnicka HM, Thiele J, Staib P, Schmitt-Graeff A, Griesshammer M, Klose J, et al. Reversal of bone marrow angiogenesis in chronic myeloid leukemia following imatinib mesylate (STI571) therapy. Blood. 2004;103(9):3549-51.

19. Denizot Y, Fixe P, Liozon E, Praloran V. Serum interleukin-8 (IL8) and IL-6 concentrations in patients with hematologic malignancies. 1996.

20. Negaard H, Iversen N, Bowitz-Lothe I, Sandset P, Steinsvik B, Østenstad B, et al. Increased bone marrow microvascular density in haematological malignancies is associated with differential regulation of angiogenic factors. Leukemia. 2009;23(1):162-9.

21. Taverna S, Amodeo V, Saieva L, Russo A, Giallombardo M, De Leo $\mathrm{G}$, et al. Exosomal shuttling of miR-126 in endothelial cells modulates adhesive and migratory abilities of chronic myelogenous leukemia cells. Mol Cancer. 2014;13(1):1-15.

22. Muller WA. Mechanisms of transendothelial migration of leukocytes. Circul Res. 2009;105(3):223-30.

23. Rudini N, Dejana E. Adherens junctions. Curr Biol. 2008;18(23):R1080-R2.

24. Taverna S, Flugy A, Saieva L, Kohn EC, Santoro A, Meraviglia $S$, et al. Role of exosomes released by chronic myelogenous leukemia cells in angiogenesis. Int J Cancer. 2012;130(9):203343.

25. Mineo M, Garfield SH, Taverna S, Flugy A, De Leo G, Alessandro R, et al. Exosomes released by K562 chronic myeloid leukemia cells promote angiogenesis in a Srcdependent fashion. Angiogenesis. 2012:15(1):33-45.

26. Mitra SK, Hanson DA, Schlaepfer DD. Focal adhesion kinase: in command and control of cell motility. Nat Rev Mol Cell Biol. 2005;6(1):56-68.

27. Roma-Rodrigues C, Fernandes AR, Baptista PV. Counteracting the effect of leukemia exosomes by antiangiogenic gold nanoparticles. Int J Nanomedicine. 2019;14:6843.

28. $\mathrm{Hu}$ G, Drescher K, Chen X. Exosomal miRNAs: Biological Properties and Therapeutic Potential. Front Genet. 2012;3(56).

29. Feng D-Q, Huang B, Li J, Liu J, Chen X-M, Xu Y-M, et al. Selective miRNA expression profile in chronic myeloid leukemia K562 cell-derived exosomes. Asian Pacific J Cancer Prevent. 2013;14(12):7501-8.

30. Tadokoro H, Umezu T, Ohyashiki K, Hirano T, Ohyashiki JH. Exosomes derived from hypoxic leukemia cells enhance tube formation in endothelial cells. J Biologic Chem. 2013;288(48):34343-51.

31. Umezu T, Ohyashiki K, Kuroda M, Ohyashiki J. Leukemia cell to endothelial cell communication via exosomal miRNAs. Oncogene. 2013;32(22):2747-55.

32. Fish JE, Santoro MM, Morton SU, Yu S, Yeh R-F, Wythe JD, et al. miR-126 regulates angiogenic signaling and vascular integrity. Dev cell. 2008;15(2):272-84.

33. Harris TA, Yamakuchi M, Ferlito M, Mendell JT, Lowenstein CJ. MicroRNA-126 regulates endothelial expression of vascular cell adhesion molecule 1. Proc Nat Aca Sci. 2008;105(5):1516-21.

34. Bozzuto G, Ruggieri P, Molinari A. Molecular aspects of tumor cell migration and invasion. Ann Ist Super Sanita. 2010;46:66-80.

35. Liu Y, Cao X. Immunosuppressive cells in tumor immune escape and metastasis. J Mol Med. 2016;94(5):509-22.
36. Raimondo S, Saieva L, Corrado C, Fontana S, Flugy A, Rizzo A, et al. Chronic myeloid leukemia-derived exosomes promote tumor growth through an autocrine mechanism. Cell Commun. Signal. 2015;13(1):1-12.

37. Ikushima H, Miyazono K. TGFbeta signalling: a complex web in cancer progression. Nat Rev Cancer. 2010;10(6):415-24.

38. Suthiphongchai T, Promyart P, Virochrut S, Tohtong R, Wilairat $P$. Involvement of ERK1/2 in invasiveness and metastatic development of rat prostatic adenocarcinoma. Oncol Res 2003;13(5):253-9.

39. Sethi G, Ahn KS, Aggarwal BB. Targeting nuclear factor-kB activation pathway by thymoquinone: role in suppression of antiapoptotic gene products and enhancement of apoptosis. Mol Cancer Res. 2008;6(6):1059-70.

40. Corrado C, Raimondo S, Saieva L, Flugy AM, De Leo G, Alessandro R. Exosome-mediated crosstalk between chronic myelogenous leukemia cells and human bone marrow stromal cells triggers an interleukin 8-dependent survival of leukemia cells. Cancer Lett. 2014;348(1-2):71-6.

41. Edlinger L, Berger-Becvar A, Menzl I, Hoermann G, Greiner G, Grundschober E, et al. Expansion of BCR/ABL 1+ cells requires PAK 2 but not PAK 1. Br J Haematol. 2017;179(2):229-41.

42. Morrison SJ, Scadden DT. The bone marrow niche for haematopoietic stem cells. Nature. 2014;505(7483):327-34.

43. Borgovan T, Crawford L, Nwizu C, Quesenberry P. Stem cells and extracellular vesicles: biological regulators of physiology and disease. Am J Physiol Cell Physiol. 2019;317(2):C155-C66.

44. Corrado C, Saieva L, Raimondo S, Santoro A, De Leo G, Alessandro R. Chronic myelogenous leukaemia exosomes modulate bone marrow microenvironment through activation of epidermal growth factor receptor. J Cell Mol Med. 2016;20(10):1829-39.

45. Kerpedjieva SS, Kim DS, Barbeau DJ, Tamama K. EGFR ligands drive multipotential stromal cells to produce multiple growth factors and cytokines via early growth response-1. Stem cells Dev. 2012;21(13):2541-51.

46. Liu Z-c, Chen X-h, Song H-X, Wang H-s, Zhang G, Wang H, et al. Snail regulated by PKC/GSK-3 $\beta$ pathway is crucial for EGFinduced epithelial-mesenchymal transition (EMT) of cancer cells. Cell Tissue Res 2014;358(2):491-502.

47. Jafarzadeh N, Safari Z, Pornour M, Amirizadeh N, Forouzandeh Moghadam M, Sadeghizadeh M. Alteration of cellular and immune-related properties of bone marrow mesenchymal stem cells and macrophages by K562 chronic myeloid leukemia cell derived exosomes. J Cell Physiol. 2019;234(4):3697-710.

48. Tabe Y, Konopleva M. Advances in understanding the leukaemia microenvironment. Br J Haematol. 2014;164(6):767-78.

49. Vianello F, Villanova F, Tisato V, Lymperi S, Ho K-K, Gomes AR, et al. Bone marrow mesenchymal stromal cells non-selectively protect chronic myeloid leukemia cells from imatinib-induced apoptosis via the CXCR4/CXCL12 axis. Haematologica. 2010;95(7):1081-9.

50. D’Amico L, Mahajan S, Capietto A-H, Yang Z, Zamani A, Ricci B, et al. Dickkopf-related protein 1 (Dkk1) regulates the accumulation and function of myeloid derived suppressor cells in cancer. J Exp Med. 2016;213(5):827-40.

51. Asem MS, Buechler S, Wates RB, Miller DL, Stack MS. Wnt5a signaling in cancer. Cancers. 2016;8(9):79.

52. Gallipoli P, Pellicano F, Morrison H, Laidlaw K, Allan EK, Bhatia $\mathrm{R}$, et al. Autocrine TNF- $\alpha$ production supports CML stem and progenitor cell survival and enhances their proliferation. Blood. 2013;122(19):3335-9.

53. Zhou X, Li Z, Zhou J. Tumor necrosis factor $\alpha$ in the onset and progression of leukemia. Exp Hematol. 2017;45:17-26.

54. Ren G, Zhang L, Zhao X, Xu G, Zhang Y, Roberts Al, et al. Mesenchymal stem cell-mediated immunosuppression occurs via concerted action of chemokines and nitric oxide. Cell stem cell. 2008;2(2):141-50.

55. Sangwan L, Kumar R, Peter R, Arun P. Evaluation of nitric oxide levels in chronic myeloid leukemia. Am J innov res appl sci. 2014;2:1-5.

56. Williams EL, Djamgoz MB. Nitric oxide and metastatic cell behaviour. Bioessays. 2005;27(12):1228-38.

57. Gao X, Wan Z, Wei M, Dong Y, Zhao Y, Chen X, et al. Chronic myelogenous leukemia cells remodel the bone marrow niche via 
exosome-mediated transfer of miR-320. Theranostics. 2019;9(19):5642.

58. Xishan Z, Ziying L, Jing D, Gang L. MicroRNA-320a acts as a tumor suppressor by targeting $B C R / A B L$ oncogene in chronic myeloid leukemia. Sci Rep. 2015;5(1):1-11.

59. Iervolino A, Santilli G, Trotta R, Guerzoni C, Cesi V, Bergamaschi $A$, et al. hnRNP A1 nucleocytoplasmic shuttling activity is required for normal myelopoiesis and BCR/ABL leukemogenesis. Mol cell biol 2002;22(7):2255-66.

60. Corrado C, Flugy AM, Taverna S, Raimondo S, Guggino G, Karmali R, et al. Carboxyamidotriazole-orotate inhibits the growth of imatinib-resistant chronic myeloid leukaemia cells and modulates exosomes-stimulated angiogenesis. PLoS One. 2012;7(8):e42310.

61. Yang K, Fu L-w. Mechanisms of resistance to BCR-ABL TKls and the therapeutic strategies: A review. Crit Rev Oncol Hematol. 2015;93(3):277-92.

62. Min Q-H, Wang X-Z, Zhang J, Chen Q-G, Li S-Q, Liu X-Q, et al. Exosomes derived from imatinib-resistant chronic myeloid leukemia cells mediate a horizontal transfer of drug-resistant trait by delivering miR-365. Exp Cell Res. 2018 Jan 15;362(2):386393.

63. Hsu D-H, Paz P, Villaflor G, Rivas A, Mehta-Damani A, Angevin $\mathrm{E}$, et al. Exosomes as a Tumor Vaccine: Enhancing Potency Through Direct Loading of Antigenic Peptides. J Immunother.2003;26(5):440-50.

64. Delcayre A, Le Pecq J-B. Exosomes as novel therapeutic nanodevices. Curr Opin Mol Ther. 2006;8(1):31-8.

65. Beauvillain C, Juste MO, Dion S, Pierre J, Dimier-Poisson I. Exosomes are an effective vaccine against congenital toxoplasmosis in mice. Vaccine. 2009;27(11):1750-7.
66. Prado N, Marazuela EG, Segura E, Fernández-García H, Villalba $M$, Théry $C$, et al. Exosomes from Bronchoalveolar Fluid of Tolerized Mice Prevent Allergic Reaction. J Immunol 2008; 181(2):1519-25.

67. Kim S-H, Lechman ER, Bianco N, Menon R, Keravala A, Nash J, et al. Exosomes Derived from IL-10-Treated Dendritic Cells Can Suppress Inflammation and Collagen-Induced Arthritis. J Immunol $2005 ; 174(10): 6440-8$.

68. Lai RC, Yeo RWY, Tan KH, Lim SK. Exosomes for drug delivery - a novel application for the mesenchymal stem cell. Biotechnol Adv 2013;31(5):543-51.

69. J O'Loughlin A AWC, JA Wood M. Exosomes and the emerging field of exosome-based gene therapy. Curr Gene Ther . 2012 Aug;12(4):262-74.

70. Dong $\mathrm{Y}$, Lin $\mathrm{Y}$, Gao X, Zhao Y, Wan Z, Wang H, et al. Targeted blocking of miR328 lysosomal degradation with alkalized exosomes sensitizes the chronic leukemia cells to imatinib. Appl Microbiol Biotechnol. 2019;103(23-24):9569-9582.

71. Taverna S, Giallombardo M, Pucci M, Flugy A, Manno M, Raccosta S, et al. Curcumin inhibits in vitro and in vivo chronic myelogenous leukemia cells growth: a possible role for exosomal disposal of miR-21. Oncotarget. 2015;6(26):21918-33

72. Bellavia D, Raimondo S, Calabrese G, Forte S, Cristaldi M, Patinella $A$, et al. Interleukin 3 - receptor targeted exosomes inhibit in vitro and in vivo Chronic Myelogenous Leukemia cell growth. Theranostics. 2017;7(5):1333-45.

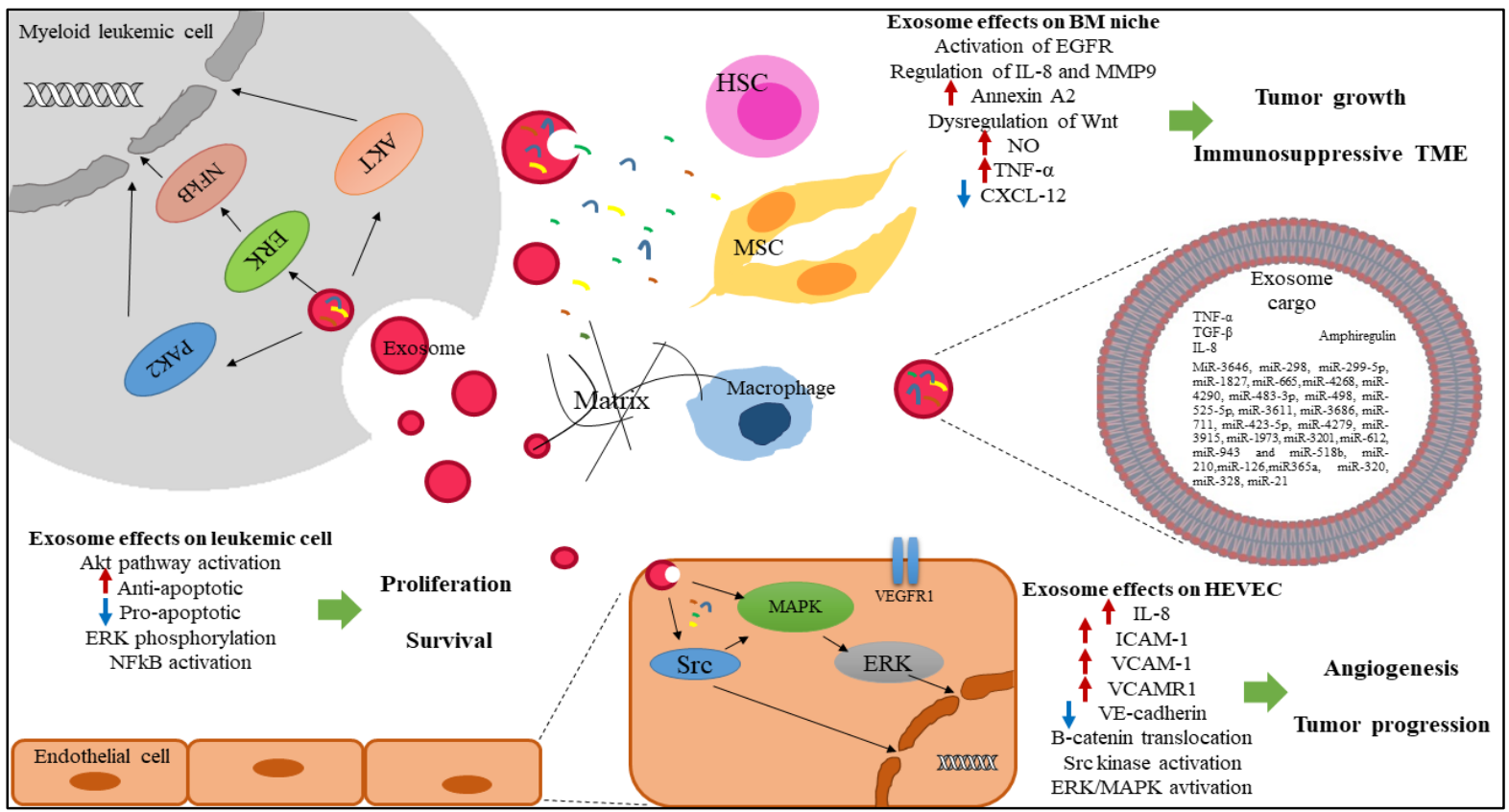

Figure 1: CML-derived exosomes effects on the disease progression. CML cells derived exosomes could affect other tumor cells and TME cell's properties. Exosome can promote angiogenesis by inducing IL-8 expression and adhesion molecules (VCAM-1, ICAM-1) along with decreasing $\mathrm{V}$-cadherin and translocating $\mathrm{b}$-catenin in endothelial cells. Exosomes also increase tumor proliferation and survival by elevating anti-apoptotic and reducing pro-apoptotic molecules. TGF- $\beta$ - enriched exosomes induce proliferation and differentiation of leukemic cells. BM stromal cells' exposure to exosomes could elevate annexinA2 expression on their surface and promote leukemic cell adhesion on the stroma. Exosomes could decrease CXCL12 release by MSCs in BM niche which is associated with HSCs support reduction and leukemic cell growth induction. Exosomes can provide an immunosuppressive TME through the accumulation of MDSCs and macrophage polarization to M2 phenotype. 